\title{
Validation of a Temperature Emissivity Separation Hybrid Method from Airborne Hyperspectral Scanner Data and Ground Measurements in the SEN2FLEX Field Campaigns
}

\author{
L. F. Peres ${ }^{1,2}$, J. A. Sobrino ${ }^{3}$, R. Libonati ${ }^{2,1}$, J. C. Jiménez-Muñoz ${ }^{3}$, M. Romaguera ${ }^{3}$, C. \\ C. DaCamara ${ }^{2}$
}

${ }^{I}$ Centro de Previsão do Tempo e Estudos Climáticos, Instituto Nacional de Pesquisas

Espaciais, Brazil

${ }^{2}$ University of Lisbon, Centro de Geofisica da Universidade de Lisboa, IDL, Portugal

${ }^{3}$ Universitat de València, Spain

lperes@cptec.inpe.br

\begin{abstract}
This paper presents an assessment of the performance of a hybrid method that allows a simultaneous retrieval of land-surface temperature (LST) and emissivity (LSE) from remote-sensed data. The proposed method is based on a synergistic usage of the split-window (SW) and the two-temperature method (TTM) and combines the advantages of both methods while mitigating their drawbacks. The method was implemented for Airborne Hyperspectral Scanner (AHS) thermal channels $76(10.56 \mu \mathrm{m})$ and $78(11.72 \mu \mathrm{m})$, which was flown over the Barrax test site (Albacete, Spain) in the framework of the SENtinel-2 and FLuorescence EXperiment (SEN2FLEX) field campaigns in the third week of July 2005. A set of radiometric measurements were performed in the thermal infrared region for different surface types, e.g., bare soil, water body, corn, wheat, grass. These measurements include surface temperature in coincidence with aircraft overpasses, thermal angular measurements using a goniometric motorized system, thermal imagery with two thermal cameras and emissivity measurements by means of the Box Method.
\end{abstract}

\section{INTRODUCTION}

Land-surface temperature (LST) is an importan parameter for understanding land-atmosphere interactions because it is sensitive to the partitioning of energy and mass fluxes at the Earth's surface. Instruments on-board Earth observation satellites and working in the thermal infrared (TIR) spectrum are currently expected to provide measurements of LST on a global basis with uniformity and continuity at spatial and temporal resolutions that are suited for most modelling applications (Viterbo, 2002; Bouyssel, 2002).

Problems encountered in estimating LST from TIR remote sensing data mainly relate to the fact that radiance measured is not only affected by LST, but also by land-surface emissivity (LSE), as well as by the thermal structure and composition of the atmosphere. Therefore, an accurate retrieval of LST from space data requires a proper characterization of the atmospheric influence as well as a distinction between the effects of LST and LSE, which is no possible solely based on observations. Without any a priori information, it is impossible to recover both parameters uniquely, and the developed techniques differ according to the required closing assumptions. For instance, split-window (SW) algorithms assume that LSE is known a priori and the task turns easier in this case because the nature of the problem is deterministic and the methods basically reduce to performing an atmospheric correction for known LSE, which is based on the differential absorption in two adjacent TIR bands within the same atmospheric window. Several formulations have been derived with different levels of refinement, where LST is in general expressed by means of linear combinations of brightness temperature (BT). Besides their simplicity and computational efficiency the main advantage of SW algorithms is that radiosounding measurements are not required to perform the atmospheric correction. However, different authors (e.g., Becker, 1987; Wan and Dozier, 1996) have shown that the main drawback of SW algorithms is that large errors on LST may arise due to uncertainties in LSE.

On the other hand, the two-temperature method (TTM) does not strictly assume an a priori knowledge of LSE allowing a simultaneous retrieval of LST and LSE if surface is observed at least at two different temperatures and if it is assumed that LSE does not change between observations. Accordingly, such type of methods are usually referred to in the literature as 
emissivity-temperature separation algorithms. The common characteristics and drawbacks of such approaches are that they require accurate temperature and humidity atmospheric profiles as inputs to a radiative transfer model (RTM) in order to perform the atmospheric correction.

In order to circumvent the main drawbacks of both above-mentioned methods, we have proposed a new hybrid procedure that is based on a synergistic use of SW and TTM, and the goal is to combine the most attractive features of both methods while mitigating some of the pitfalls.

The objective of this work is to assess the accuracy of the developed hybrid method and compare its performance with a SW algorithm. The study was performed using data from the Airborne Hyperspectral Scanner (AHS) and ground-based measurements acquired in the framework of the SENtinel-2 and FLuorescence EXperiment (SEN2FLEX) field campaigns.

\section{FIELD DATA}

A set of radiometric measurements was performed in the TIR region concurrently to the AHS flights (see Table 1) over the Barrax test site (Albacete, Spain) within the framework of the SEN2FLEX field campaigns held in July 12, 13 and 14, 2005. It is worth noting that the Barrax test site is characterised by a flat morphology and large, uniform land-use units, with differences in elevation only ranging up to $2 \mathrm{~m}$. The region consists of approximately 65\% dry and 35\% irrigated land with different types of crop fields and orchards (figure 1).

The AHS instrument is an imaging 80-band linescanner radiometer with 10 bands (71 to 80 ) in the long-wave infrared (LWIR) port covering the atmospheric window $(8.2$ to $12.9 \mu \mathrm{m})$. It is worth noting that we have solely used the AHS bands 76 $(10.56 \mu \mathrm{m})$ and $78(11.72 \mu \mathrm{m})$ since they have resulted in the best combination for the developed SW algorithm. The data presented here are from flights at an altitude of $1340 \mathrm{~m}$, yielding a pixel resolution of $3.4 \mathrm{~m}$. The atmospheric correction of the AHS thermal channels was performed based on information about the atmospheric state, namely from humidity and temperature profiles from local radiosounding measurements, used as input to MODTRAN4 (Berk et al., 2000).

Transects were realized over selected surfaces concurrently to the plane overpasses, starting half an hour before and ending half an hour after. Thermal measurements were also continuously recorded with radiometers located on fixed masts over selected areas and periods of time. LSE of representative samples was measured by means of the Box method and by using the CIMEL CE312-2 radiometer together with the temperature-emissivity separation (TES) algorithm (Gillespie et al., 1999). Figure 2 shows the different parcels where the measurements where carried out and the validation was performed.

Table 1. Date and time (UTM) of the AHS flights

\begin{tabular}{cccccc}
\hline \multicolumn{2}{c}{ July 12} & \multicolumn{2}{c}{ July 13} & \multicolumn{2}{c}{ July 14} \\
\hline $12: 21$ & $22: 32$ & $08: 15$ & $12: 09$ & $08: 23$ & $12: 25$ \\
\hline
\end{tabular}

\section{METHOD}

TTM allows for a simultaneous retrieval of LST and LSE if the surface is observed at least at two different temperatures. The method assumes that LSE does not change between observations and that atmospheric effects may be adequately estimated by means of a RTM. The radiance recorded under a zenith angle $\theta$ in a given channel $c$ may be represented by the radiative transfer equation (RTE)

$L_{R T E_{c}}(\theta)=\varepsilon_{c} B_{c}\left(T_{s}\right) \tau_{c}(\theta)+L_{c}^{\uparrow}(\theta)+L_{c}^{\downarrow}\left(1-\varepsilon_{c}\right) \tau_{c}(\theta)$

where $\varepsilon_{c}, B_{c}\left(T_{s}\right), \tau_{c}(\theta), L_{c}^{\dagger}(\theta)$ and $L_{c}^{\downarrow}$ respectively denote the LSE, the emitted radiance given by Planck's function for the surface temperature $T_{s}$, the atmospheric transmittance, and the atmospheric upward and downward radiances. Assuming that a pair of observations is available (performed at times $t_{1}$ and $t_{2}$ in the two AHS channels 76 and 78) then the 4 unknown surface parameters (i.e., $T_{s}$ at times $t_{1}$ and $t_{2}$, and $\varepsilon$ in channels 76 and 78) may be obtained by solving a system of 4 equations, each one of the form given by Eq. (1). Following previous studies (Faysash and Smith, 2000; Peres and DaCamara, 2004), a quasiNewton optimization method (Gill et al., 1983) was used in order to find the unknowns that minimize the cost function $f$ given by the sum of squared differences between radiances $L_{O B S}$ (observed radiances) and $L_{\text {RTE }}$ (computed radiances by means of the RTE model as given by Eq. (1))

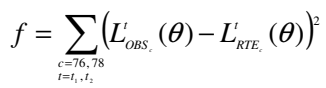

The hybrid procedure we have proposed consists in combining the use of a priori LSE estimates from surface emissivity maps (Peres and DaCamara, 2005) together with LST estimates as obtained from a SW algorithm (Sobrino and Raissouni., 2000). The aim is to define narrower and more reliable ranges of admissible solutions before applying TTM (figure 3) and the rationale is to increase the efficiency of TTM 
from a computational point of view and especially to improve the quality of LST retrievals over areas where LSE is not well known a priori.

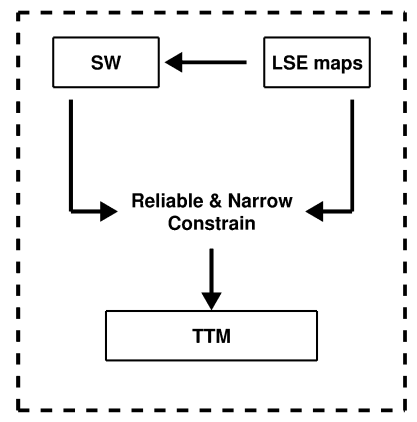

Figure 3. Schematic representation of the hybric method

In order to test the performance of the hybrid method we have assumed a general case where LSE is not well known a priori in the study area. Accordingly, we have assigned laboratory measurements of LSE (as obtained from the Johns Hopkins University (JHU) Spectral Library) to the GLC2000 Land Cover map (see figure 1), which has a $1-\mathrm{km}$ nominal spatial resolution. GLC2000 classifies the whole area of the Barrax test site as Cultivated/Managed Areas and taking into account that soils of the molisol-type tend to be base rich and quite fertile, being the best agricultural soil, this class was characterized by the following samples and weights: $50 \%$ of green grass + $50 \%$ of molisols. The prescribed LSE values for channels 76 and 78 are 0.976 and 0.980 , respectively.

In order to obtain a priori estimates on LST from SW, we have implemented the SW algorithm of Sobrino and Raissouni (2000) for AHS thermal channels 76 and 78

$$
\begin{aligned}
T_{s}= & a_{0}+a_{1} T_{76}+a_{2}\left(T_{76}-T_{78}\right)+a_{3}\left(T_{76}-T_{78}\right)^{2} \\
& +a_{4}(1-\mathcal{E})+a_{5} \Delta \mathcal{E}
\end{aligned}
$$

where $T_{76}\left(T_{78}\right)$ is the brightness temperature in channel 76 (78), $\varepsilon=\left(\varepsilon_{76}+\varepsilon_{78}\right) / 2$ is the average emissivity in channels 76 and $78, \Delta \varepsilon=\left(\varepsilon_{76}-\varepsilon_{78}\right)$ is the emissivity difference between the two channels, and $a_{k}(k=0$ to 5$)$ are SW coefficients estimated by means of regression analysis of simulated observed radiances as obtained with MODTRAN4. Accordingly, the following cases were considered in the simulation a) 165 profiles from TIGR database; b) LST varying around the air temperature at 2-meter
( $T_{a}$ ) from $T_{a}-10.0 \mathrm{~K}$ to $T_{a}+15.0 \mathrm{~K}$ in steps of 5.0 $\mathrm{K}$; c) $\varepsilon$ varying from 0.90 to 0.99 in steps of 0.01 , and $\Delta \varepsilon$ varying from -0.01 to 0.01 in steps of 0.01 ; and d) 8 satellite zenith angles (SZA) covering a range of values from nadir to $60.0^{\circ}$.

Finally, we have applied the hybrid method according to the following three steps: 1) use as input LSE $_{-M}$ directly form LSE map and apply SW to obtain LST_sw; 2) define the set of admissible solution for TTM as LST_Sw $\pm 3.0 \mathrm{~K}$ and $\mathrm{LSE}_{-\mathrm{M}} \pm$ 0.02 where $3.0 \mathrm{~K}$ and 0.02 represent the assumed uncertainties of SW and LSE maps; and 3) search LST and LSE combination that minimizes Eq. (2).

\section{RESULTS}

In order to assess the improvements on LST and LSE estimations allowed by the proposed hybrid method, we have considered the following two cases: 1) SW is used alone and 2) the new hybrid method is applied. Results respecting to the single usage of SW are shown in Figure 4 for AHS flights, whereas those obtained using the hybrid method are shown in Figures 5 and 6 .

Table 2 shows values of bias, standard deviation (STD) and root-mean square error (RMSE) between ground measurements and derived LST using each method. The hybrid method has provided LST values with bias (RMSE) of $0.8 \mathrm{~K}(1.9 \mathrm{~K})$, whereas for LSE the corresponding obtained values were -0.009 $(0.015)$. These figures point out the better performance of the hybrid method and are worth being compared with those obtained when using SW alone, namely the values of $2.7 \mathrm{~K}(3.4 \mathrm{~K})$ for the bias (RMSE).

Table 2. Result for all sites and scenes

\begin{tabular}{ccccc}
\hline Method & Parameter & Bias & STD & RMSE \\
\hline \multirow{2}{*}{ Hybrid } & LSE & -0.009 & 0.012 & 0.015 \\
& LST (K) & 0.8 & 1.8 & 1.9 \\
SW & LST (K) & 2.7 & 2.1 & 3.4 \\
\hline
\end{tabular}

\section{CONCLUSIONS}

We have validated a new hybrid method based on a synergistic usage of SW and TTM, which combines the attractive features of both methods while mitigating some of their drawbacks. The hybrid method was tested and compared with a SW algorithm using data from AHS and ground-based measurements acquired in the framework of the SEN2FLEX field campaigns. We have shown that the hybrid method is able to provide better estimates of LST, with values of bias (RMSE) of the order of $0.8 \mathrm{~K}(1.9 \mathrm{~K})$, i.e. about one third (one half) of the corresponding values of 2.7 
$\mathrm{K}$ for bias (3.4 K for RMSE) that were obtained when using the SW algorithm.

\section{REFERENCES}

Becker, F., 1987, The impact of spectral emissivity on the measurement of land surface temperature from satellite. International Journal of Remote Sensing, 8, 1509-1522.

Berk, A., Anderson, G. P., Acharya, P. K., Chetwynd, J. H., Bernstein, L. S., Shettle, E. P., Matthew, M W., and Alder-Golden, S. M., 2000, MODTRAN4 version 2 user's manual. Air Force Research Laboratory, Space Vehicles Directorate, Air Force Material Command, Hanscom AFB, MA 01731-3010

Bouyssel, F., 2002, NWP needs: The Météo-France point of view. Presented at: LSA SAF Training Workshop, Lisbon, Portugal, 8-10 July.

Faysash, A., and Smith, E. A., 2000, Simultaneous retrieval of diurnal to seasonal surface temperatures and emissivities over SGP ARMCART site using GOES split window. Journal of Applied Meteorology, 39, 971-982.

Gill, P. E., Murray, W., and Wright, M. H, 1983 , Practical optimization. New York: Academic Press.

Gillespie, A. R., Rokugawa, S., Hook, S. J., Matsunaga, T., and Kahle, A. B., 1999 Temperature/emissivity separation algorithm theoretical basis document. Version 2.4. Prepared under NASA Contract NAS5-31372.

Peres, L. F., and DaCamara, C. C., 2004, Land surface temperature and emissivity estimation based on the Two-Temperature Method: Sensitivity analysis using simulated MSG/SEVIRI data Remote Sensing of Environment, 91, 377-389.

Peres, L. F., and DaCamara, C. C., 2005, Emissivity maps to retrieve land-surface temperature from MSG/SEVIRI Data. IEEE Transactions on Geoscience and Remote Sensing, 45, 1834-1844.

Sobrino, J.A and Raissouni, N., 2000, Toward remote sensing methods for land cover dynamic monitoring. Application to Morocco. International Journal of Remote Sensing, 20(2), $353-366$

Viterbo, P., 2002, NWP needs: The ECMWF (et al.) perspective. Presented at: LSA SAF Training Workshop, Lisbon, Portugal, 8-10 July.

Wan, Z., and Dozier, J., 1996, A generalised splitwindow algorithm for retrieving land-surface temperature from space. IEEE Transactions on Geoscience and Remote Sensing, 34, 892-905.

\section{COLOR FIGURES}

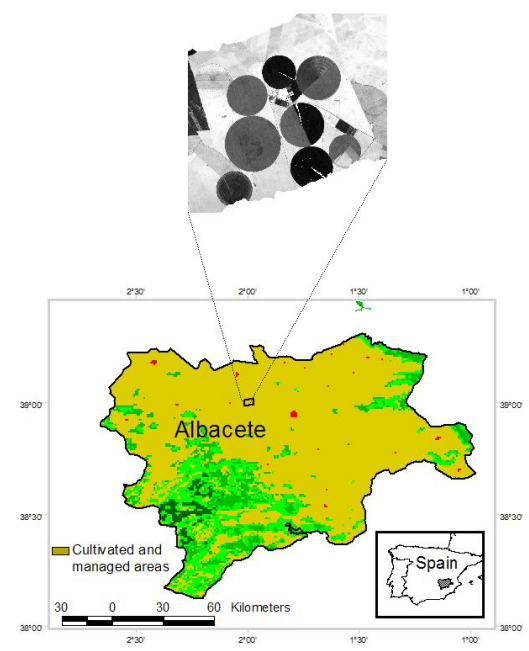

Figure 1. The Barrax test site in the province of Albacete, respective location in Spain, and main vegetation types, as defined by the GLC2000 Land Cover map.

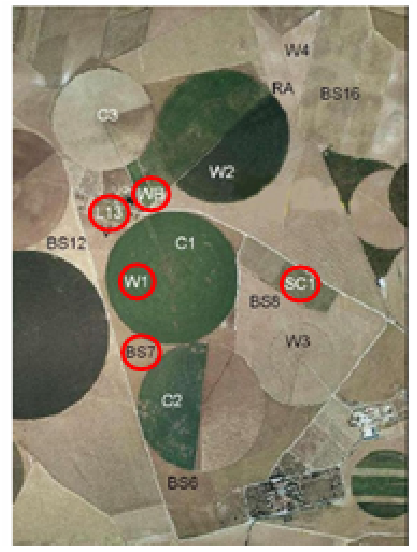

Figure 2. Location and land cover types of parcels where comparisons were performed. L13 is grass, WB is water, W1 is wheat, BS7 is bare soil and SC1 is corn. 

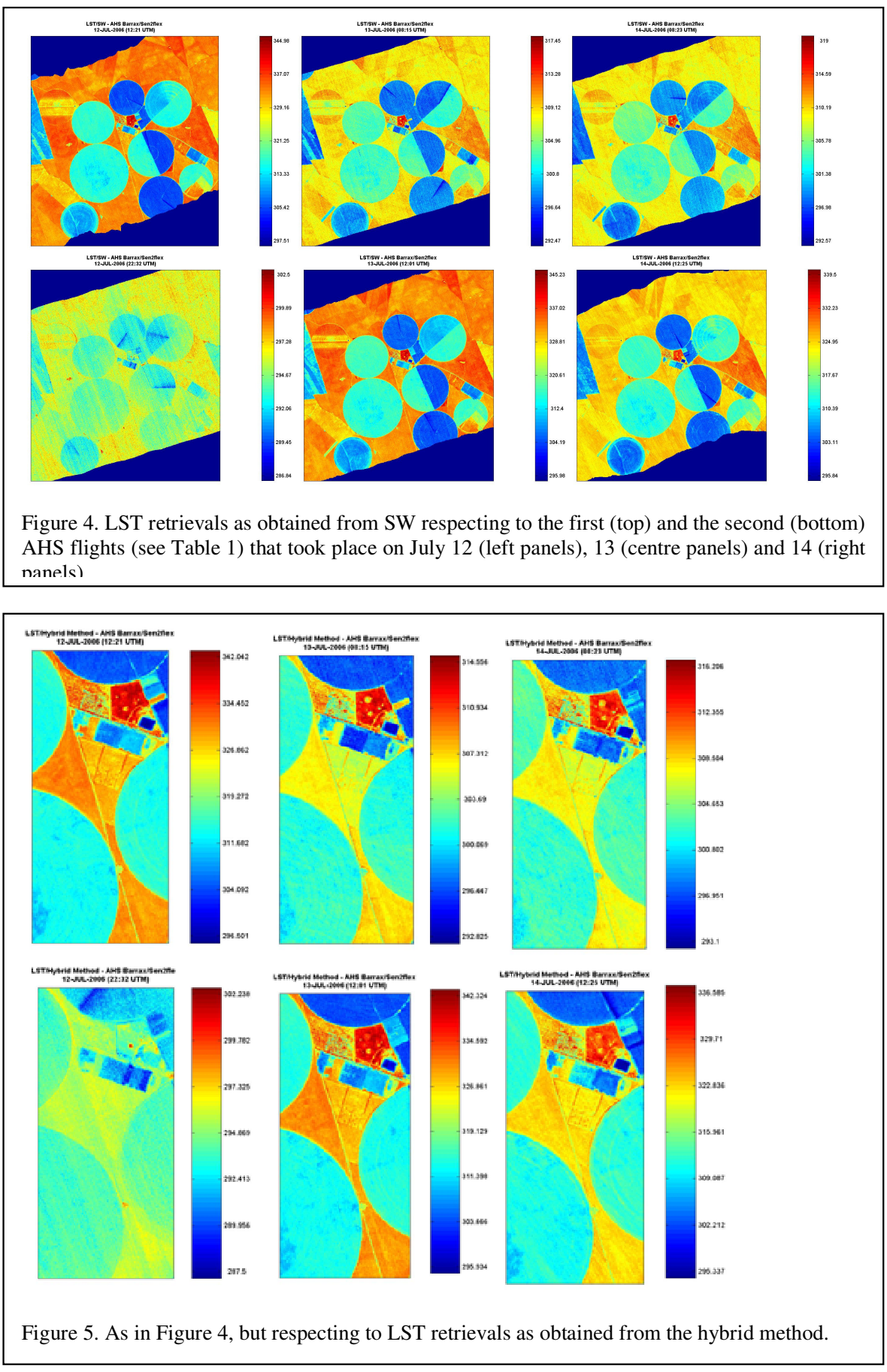
INPE ePrint: sid.inpe.br/mtc-m17@80/2007/04.20.18.34 v2 2007-07-31

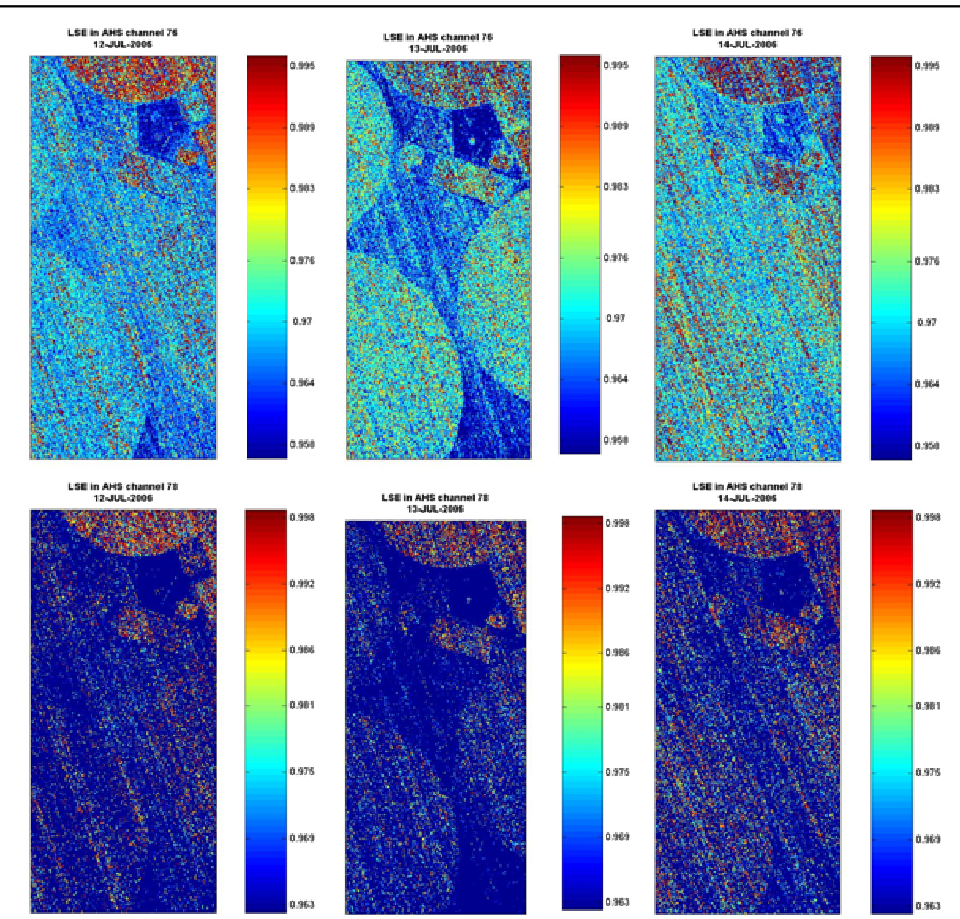

Figure 6. LSE retrievals as obtained from the hybrid method respecting to AHS channels 76 (top) and 78 (bottom) and to the flights of July 12 (left panels), 13 (centre panels) and 14 (right panels). 